\title{
Identification of Subtypes and a Prognostic Gene Signature in Colon Cancer Using Cell Differentiation Trajectories
}

\author{
Renshen Xiang ${ }^{1,2+}$, Jincheng $\mathrm{Fu}^{1,2 t}$, Yuhang $\mathrm{Ge}^{1}$, Jun $\mathrm{Ren}^{1,2}$, Wei Song ${ }^{1,2}$ and $\mathrm{TaO}_{\mathrm{Fu}}{ }^{1 *}$ \\ ${ }^{1}$ Department of Gastrointestinal Surgery II, Renmin Hospital of Wuhan University, Wuhan, China, ${ }^{2}$ Central Laboratory, Renmin \\ Hospital of Wuhan University, Wuhan, China
}

OPEN ACCESS

Edited by:

Hernandes F. Carvalho,

State University of Campinas, Brazil

Reviewed by:

Eduardo Vicente,

São Paulo State University, Brazil

Xinwei Han,

Zhengzhou University, China

*Correspondence:

Tao Fu

tfu001@whu.edu.cn

these authors have contributed equally to this work

Specialty section:

This article was submitted to

Molecular and Cellular Oncology,

a section of the journal

Frontiers in Cell and Developmental

Biology

Received: 05 May 2021 Accepted: 17 November 2021 Published: 13 December 2021

Citation:

Xiang R, Fu J, Ge Y, Ren J, Song W and Fu T (2021) Identification of

Subtypes and a Prognostic Gene Signature in Colon Cancer Using Cell

Differentiation Trajectories. Front. Cell Dev. Biol. 9:705537. doi: 10.3389/fcell.2021.705537
Research on the heterogeneity of colon cancer (CC) cells is limited. This study aimed to explore the CC cell differentiation trajectory and its clinical implication and to construct a prognostic risk scoring (RS) signature based on CC differentiation-related genes (CDRGs). Cell trajectory analysis was conducted on the GSE148345 dataset, and CDRG-based molecular subtypes were identified from the GSE39582 dataset. A CDRG-based prognostic RS signature was constructed using The Cancer Genome Atlas as the training set and GSE39582 as the validation set. Two subsets with distinct differentiation states, involving 40 hub CDRGs regulated by $Y Y 1$ and EGR2, were identified by single-cell RNA sequencing data, of which subset I was related to hypoxia, metabolic disorders, and inflammation, and subset II was associated with immune responses and ferroptosis. The CDRG-based molecular subtypes could successfully predict the clinical outcomes of the patients, the tumor microenvironment status, the immune infiltration status, and the potential response to immunotherapy and chemotherapy. A nomogram integrating a five-CDRG-based RS signature and prognostic clinicopathological characteristics could successfully predict overall survival, with strong predictive performance and high accuracy. The study emphasizes the relevance of CC cell differentiation for predicting the prognosis and therapeutic response of patients to immunotherapy and chemotherapy and proposes a promising direction for CC treatment and clinical decision-making.

Keywords: colon cancer, cell differentiation trajectory, immunotherapy, chemotherapy, risk scoring

\footnotetext{
Abbreviations: CC, colon cancer (CC); CDRGs, CC differentiation-related genes; FDR, false discovery rate; GDSC, genomics of drug sensitivity in cancer; GEO, Gene Expression Omnibus; GO, Gene Ontology; ICG, immune checkpoint gene; KEGG, Kyoto Encyclopedia of Genes and Genomes; OS, overall survival; PCA, principal component analysis; PC, principal component; ROC, receiver operating characteristic; RS, risk scoring; scRNA-seq, single-cell RNA sequencing; ssGSEA, single-sample gene set enrichment analysis; TCGA, the cancer genome atlas; TF, transcription factor; tSNE, t-distributed stochastic neighbor embedding; WGCNA, weighted correlation network analysis.
} 


\section{INTRODUCTION}

Colon cancer (CC) is the third most frequently diagnosed malignant tumor worldwide, with 945,000 newly diagnosed cases per year, accounting for $9.4 \%$ of all cancer cases, and with 492,000 cancer deaths per year, accounting for $7.9 \%$ of total deaths (Parkin, 2001; Bray et al., 2018). According to the SEER database, the major histological types of CC are adenocarcinoma, mucinous adenocarcinoma, and signet ring cell carcinoma, which account for $90.1 \%, 8.7 \%$, and $1.2 \%$ of CC cases, respectively, and the 5-year survival rates are $72.2 \%$, $67.4 \%$, and $41.4 \%$, respectively. The clinical manifestations and prognosis of different pathological types vary greatly (Deng et al., 2006; Verhulst et al., 2012; Nitsche et al., 2013; Wang et al., 2016). Currently, primary treatment modalities for CC include chemotherapy along with surgery, radiotherapy, and, more recently, targeted therapy for patients with advanced late-stage CC, which significantly prolongs the overall survival (OS) and improves the prognosis of patients. However, the multidrug resistance of tumor cells to chemotherapy and molecular targeted therapy has been a major obstacle in cancer research, severely limiting the efficacy of CC therapy (Holohan et al., 2013; Li et al., 2013; Guo et al., 2017). Recently, new therapeutic methods such as immunotherapy have been gradually introduced into clinical practice (Pagès et al., 2018). Unfortunately, the OS of patients has not greatly improved, necessitating the development of novel strategies to overcome drug resistance in cancer therapy.

Intratumoral heterogeneity refers to the emergence of new gene mutations and molecular phenotypes in each filial generation after the malignant transformation of primary cells throughout continuous evolution. Therefore, a tumor is considered a mixture of different tumor cell clones (DagogoJack and Shaw, 2018). Currently, intratumoral heterogeneity is a major cause of chemoresistance; therefore, it is essential to characterize the intratumoral heterogeneity of CC cells. The development of single-cell RNA sequencing (scRNA-seq) technology has provided an effective method to reveal the characteristics of the single-cell transcriptome, opening up new avenues to investigate intratumoral heterogeneity (Lovett, 2013; Liang et al., 2014). This study aimed to analyze the cell differentiation trajectory in CC through scRNA-seq and investigate its relationship with clinical outcomes and potential responses to immunotherapy and chemotherapy, to gain new insights into the development of treatment strategies against CC and the evaluation of patient prognoses.

\section{MATERIALS AND METHODS}

\section{Data Acquisition and Processing}

The scRNA-seq data of 1,441 cells in 15 CC samples were downloaded from the GSE148345 dataset in the Gene Expression Omnibus (GEO, https://www.ncbi.nlm.nih.gov/geo/ ) database. The percentage of mitochondrial genes was calculated using the "PercentageFeatureSet" function in $\mathrm{R}$ software. The correlation between sequencing depth and length of
TABLE 1 | Clinicopathological features of patients with colon cancer.

\begin{tabular}{|c|c|c|}
\hline Variables & $\begin{array}{c}\text { GSE39582 } \\
\text { cohort }(n=585)\end{array}$ & $\begin{array}{c}\text { TCGA } \\
\text { cohort }(n=385)\end{array}$ \\
\hline \multicolumn{3}{|l|}{ Age (year) } \\
\hline Mean $\pm \mathrm{SD}^{\mathrm{a}}$ & $66.9 \pm 13.2$ & $67.0 \pm 12.8$ \\
\hline \multicolumn{3}{|l|}{ Sex } \\
\hline Female & $263(45.0)$ & $180(46.8)$ \\
\hline Male & $322(55.0)$ & 205 (53.2) \\
\hline \multicolumn{3}{|l|}{ Clinical stage } \\
\hline I & $38(6.5)$ & 66 (17.1) \\
\hline$\|$ & 271 (46.3) & 151 (39.2) \\
\hline III & $210(35.9)$ & $103(26.8)$ \\
\hline IV & 60 (10.3) & $54(14.0)$ \\
\hline Unknown & $6(1.0)$ & $11(2.9)$ \\
\hline \multicolumn{3}{|l|}{ T stage } \\
\hline $\mathrm{T} 1$ & $12(2.1)$ & $10(2.6)$ \\
\hline $\mathrm{T} 2$ & $49(8.4)$ & 68 (17.7) \\
\hline Т3 & 379 (64.8) & $263(68.3)$ \\
\hline $\mathrm{T} 4$ & 119 (20.3) & $44(11.4)$ \\
\hline Unknown & $26(4.4)$ & $0(0.0)$ \\
\hline \multicolumn{3}{|l|}{$\mathrm{N}$ stage } \\
\hline NO & $314(53.7)$ & $231(60.0)$ \\
\hline N1 & 137 (23.4) & 88 (22.9) \\
\hline $\mathrm{N} 2$ & $100(17.1)$ & 66 (17.1) \\
\hline N3 & $6(1.0)$ & $0(0.0)$ \\
\hline Unknown & $28(4.8)$ & $0(0.0)$ \\
\hline \multicolumn{3}{|l|}{ M stage } \\
\hline MO & 499 (85.3) & $286(74.3)$ \\
\hline M1 & 61 (10.4) & $54(14.0)$ \\
\hline Unknown & 25 (4.3) & $45(11.7)$ \\
\hline
\end{tabular}

astandard deviation.

mitochondrial gene sequences or total intracellular sequences was assessed by correlation analysis. Cells with an intracellular gene number $<50$, intracellular sequencing number $<3$, and mitochondrial gene fraction $>25 \%$ were excluded. After data quality control, scRNA-seq data were normalized via the "LogNormalize" algorithm. Bulk RNA-seq and relevant clinicopathological data, such as sex, age, and grade, of 39 normal and 385 CC samples in The Cancer Genome Atlas (TCGA) database (http://cancergenome.nih.gov/) and 585 CC samples in the GSE39582 dataset were used for molecular typing identification and risk scoring (RS) signature generation. In the current study, CC samples with a survival time $<30$ days, ambiguous survival status, and unclear clinicopathological features were excluded. The clinicopathological data of each sample are shown in Table 1.

\section{Dimensionality Reduction and Cell Annotation}

At a false discovery rate $(\mathrm{FDR})<0.05$, dimensions with significant separation were identified through principal component analysis (PCA) (Lall et al., 2018). Then, dimension reduction for the top 15 principal components (PCs) was performed using the t-distributed stochastic neighbor embedding (tSNE) algorithm to obtain the major clusters (Satija et al., 2015). Under the conditions of $\mid \log 2[$ fold change $(\mathrm{FC})] \mid>0.5$ and FDR $<0.05$, marker genes were screened out through differential expression 
analysis, and the top $10 \%$ of marker genes from clusters were displayed on a heat map. Clusters were annotated using the "SingleR" package in $\mathrm{R}$ and the CellMarker database (http:// biocc.hrbmu.edu.cn/CellMarker/).

\section{Cell Trajectory Analysis and Molecular Functional Analysis}

Pseudotime and cell differentiation trajectory were performed using the "Monocle" package in R (Qiu et al., 2017). With the criteria of $|\log 2(\mathrm{FC})|>1$ and $\mathrm{FDR}<0.05$, the differentially expressed genes identified from cell differentiation trajectories were designated CC differentiation-related genes (CDRGs). The "clusterProfiler" package in R was used for Kyoto Encyclopedia of Genes and Genomes (KEGG) enrichment analysis and Gene Ontology (GO) annotation for three domains, namely, biological process, cellular component, and molecular function. Hub CDRGs associated with cell differentiation trajectories were screened out through weighted correlation network analysis (WGCNA). Transcription factor (TF) enrichment analysis was conducted in the UCSC Xena platform (http://xena.ucsc.edu/).

\section{Identification of CDRG-Based Molecular Subtypes of Patients With CC From the GSE39582 Dataset}

The "ConsensusClusterPlus" package in R was used for consensus clustering of CC samples after univariate analysis (Wilkerson and Hayes, 2010). The PCA and tSNE algorithms were used to verify the stability of CC subtypes. Kaplan-Meier analysis was conducted using the "survival" package in R. Clinicopathological features were displayed on histograms using the "ggplot2" package. Differentially expressed CDRGs with FDR $<0.05$ in specific molecular subtypes were screened for exploring their expression levels within different cell differentiation trajectories.

\section{Integrated Analysis of Tumor Microenvironment Status, Immune Infiltration, and Immune Checkpoint Gene Expression Across CC Subtypes}

The immune/stromal scores and tumor purity of each sample were calculated using the "ESTIMATE" package in R. Singlesample gene set enrichment analysis (ssGSEA) was performed using the "GSVA" package to quantify the enrichment level of each sample in 29 immune gene sets (Supplementary Material S1). The fraction of 22 immune cells in each sample from the GSE39582 dataset was predicted using the CIBERSORT algorithm. Thirty-seven immune checkpoint genes (ICGs) were accessed through an extensive literature search (Wu et al., 2016; Nishino et al., 2017; Patel et al., 2017; Yang et al., 2017; Garris et al., 2018; Zhang et al., 2018; Wang et al., 2019a; Wang et al., 2019b; Han et al., 2019). Differential expression of genes across CC subtypes was assessed using the "limma" package in $\mathrm{R}$.

\section{Prediction of Chemotherapeutic Response (IC50) for CC Subtypes}

The "pRRophetic" package has been extensively used in cancerrelated studies (PMID: 33115513, 33251044, 33738339, 34532269,34335575 , and 25229481), which can employ ridge regression to estimate the half-maximum inhibitory concentration (IC50) of samples. In this study, the "pRRophetic" package was used to predict the chemotherapeutic response of each sample in the GSE39582 dataset on the basis of the Genomics of Drug Sensitivity in Cancer (GDSC) database (https://www.cancerrxgene.org/). Twelve chemotherapeutic agents were selected, namely, camptothecin, mitomycin C, doxorubicin, gemcitabine, paclitaxel, rapamycin, sorafenib, bleomycin, docetaxel, sunitinib, cisplatin, and vinblastine, to determine the response of CC cells to chemotherapy. On the basis of the GDSC training set, 10-fold cross-validation was performed to evaluate the accuracy of the prediction of chemotherapy response.

\section{Construction and Validation of RS Signature}

The bulk RNA-seq data were normalized with the $\log 2$ scale transformation method. Intersected CDRGs from TCGA and GSE39582 cohorts were identified using the "SVA" package in R. Differential expression analysis was performed in the TCGA cohort with $|\log 2(\mathrm{FC})|>1$ and FDR $<0.05$. Univariate analysis was performed for obtaining prognostic CDRGs in the TCGA cohort. With TCGA as the training set and GSE39582 as the validation set, the prognostic CDRGs were incorporated into Lasso Cox regression analysis to construct a CDRG-based RS signature. The RS of each sample was calculated as the sum of the products of CDRGs expression levels and coefficients, using the following formula:

$$
\mathrm{RS}=\sum_{i}^{k}\left(\operatorname{Exp}_{i} \times \mathrm{Coe}_{i}\right),
$$

where " $i$ " and " $k$ " represent the " $i$ th" gene and gene number, respectively. Kaplan-Meier analysis was conducted to compare the OS between the high-risk group and the low-risk group. Receiver operating characteristic (ROC) curves were plotted to evaluate the predictive performance of the RS signature.

\section{Assessment of Expression Levels of CDRGs in the RS Signature}

A total of 20 pairs of CC samples were collected from patients with CC admitted to the Renmin Hospital of Wuhan University. The study was approved by the Ethics Committee of Renmin Hospital of Wuhan University (no. WDRY 2019-K092), and an informed consent was obtained from all patients. Total RNA was extracted from CC patient tissues using TRIzol reagent (Invitrogen, Carlsbad, CA, United States), and complementary DNA was synthesized using the total RNA and a PrimeScript RT reagent kit (Takara). Quantitative PCR (qPCR) was performed using SYBR Green (Takara) on a CFX-96 instrument (Bio-Rad Laboratories, Inc., United States). The data were computed using 
TABLE 2 | List of primers.

\begin{tabular}{|c|c|}
\hline Gene & Primer sequence $\left(5^{\prime}-3^{\prime}\right)$ \\
\hline \multirow[t]{2}{*}{ ACAA2 } & Forward: AGACCCCAGCTCTCACGATT \\
\hline & Reverse: GGCTCATGCTाTCGGTTCCT \\
\hline \multirow[t]{2}{*}{ SRI } & Forward: GAGACTTGCCGGCTTATGGT \\
\hline & Reverse: TTGTCAGGGCCTTCTGCAAT \\
\hline \multirow[t]{2}{*}{ UGT2A3 } & Forward: TGGTGTITCTCTGGGGTCAC \\
\hline & Reverse: ACAGCCGAGTATTGGCTCCT \\
\hline \multirow[t]{2}{*}{ KPNA2 } & Forward: GTGATGGCTCAGTGTTCCGA \\
\hline & Reverse: GTGCAGGATTCTTGTTGCGG \\
\hline \multirow[t]{2}{*}{ MRPL37 } & Forward: AGAGAACCAAGACGAGTGCG \\
\hline & Reverse: CACCAGAACCACGGACTTGA \\
\hline
\end{tabular}

the $2^{-\Delta \Delta \mathrm{Ct}}$ method, and expression levels of target genes were normalized to those of GAPDH. The primer sequences used for qPCR in this study are listed in Table 2. The raw data of qPCR are shown in Supplementary Material S2. Subsequently, the expression levels of CDRGs at the protein level were verified using the Human Protein Atlas database (www.proteinatlas.org).

\section{Construction and Validation of a Nomogram to Predict OS}

Univariate analysis was performed in the training and validation sets to obtain prognostic variables, which were enrolled into a nomogram for predicting 3- and 5-year OS. Then, ROC and calibration curves were used to evaluate the predictive performance of the nomogram. Furthermore, the validation set was used for estimating the stability of the nomogram.

\section{RESULTS}

\section{Quality Control and Normalization of ScRNA-Seq Data}

The scRNA-seq data of 1441 CC cells in 15 samples were downloaded from the GSE148345 dataset. After performing quality control and normalization, 349 non-compliant cells were excluded, and 1,092 cells were selected for further analysis (Figure 1A). The correlation between sequencing depth and mitochondrial gene sequences was weak, with $R=$ 0.2 , which was the minimum correlation coefficient obtained according to the best screening conditions (Figure 1B). The sequencing depth was positively correlated with total intracellular sequences $(R=0.48$, Figure $1 B)$. A total of 27,480 genes were subjected to further analysis, of which 25,980 were intercellular genes with a low-mutation frequency, and 1,500 were genes with high variability (Figure 1C).

\section{Identification of Two CC Subsets by CC Cell Trajectory Analysis}

Preliminary dimensionality reduction of scRNA-seq data was performed by PCA, but no significant segregation was observed among CC cells (Figure 2A). Therefore, the top 15 PCs with significant differences were screened for further analysis (Figure 2B). According to the tSNE algorithm, 1,092 CC cells were clustered into eight clusters, 5,224 marker genes were identified, and the top $10 \%$ of marker genes into each cluster were displayed on the heat map (Figure 2C). Eight clusters were annotated on the basis of marker genes: clusters $0 / 1 / 2 / 5 / 6 / 7$ were cancer cells, cluster 3 was macrophages, and cluster 4 was epithelial cells. The results of cell trajectory analysis revealed that cluster 4 was mainly distributed in the root, containing epithelial cells. Clusters 0/3/6 were distributed in subset I (branch I), which consisted of cancer cells and macrophages. Clusters 1/2/ $5 / 7$ were located in subset II (branch II), which consisted of cancer cells (Figures 2D,E).

\section{Molecular Functional Analysis Based on CDRGs}

We identified 153 CDRGs from the root, 800 CDRGs from subset I (branch I), and 245 CDRGs from subset II (branch II). The results of GO annotation and KEGG enrichment analysis revealed that overexpression of CDRGs in root was strictly related to the regulation of nuclear mitosis (Supplementary Figures S1A,B), and low expression of CDRGs was closely associated with immune killing (Supplementary Figures S1C,D). Highly expressed CDRGs in subset I (branch I) were steadily linked to hypoxia and inflammatory responses (Supplementary Figures S2A,B), and lowly expressed CDRGs were correlated with energy metabolic disorders (Supplementary Figures S2C,D). The upregulated CDRGs in subset II (branch II) were profoundly associated with cellular drug metabolism and immune responses (Supplementary Figures S2A,B), whereas the downregulated CDRGs were strongly correlated with ferroptosis and catabolism (Supplementary Figures S3C,D).

\section{Identification of Hub CDRGs in Two Subsets}

On the basis of WGCNA, 13 modules were generated with soft threshold $=6$ (Figure 2F), of which three modules were positively correlated with subset I (branch I), whereas nine modules were positively associated with subset II (branch II) (Figure 2G). In this study, the magenta module and the blue module were regarded as core modules that regulated cell differentiation trajectories, and hub CDRGs in two core modules are shown in Supplementary Material S3. The results of TF enrichment analysis showed that YY1 and EGR2 were the key TFs regulating the transcription of hub CDRGs (Figure 2H).

\section{Identification of CDRG-Based Molecular Subtypes of Patients With CC From the GSE39582 Dataset}

Prognostic CDRG-based consensus clustering analysis was performed on the GSE39582 dataset, and two molecular subtypes, including all the CC samples, were generated at a clustering threshold of $K=2$ (Figure 3A). The PCA and tSNE algorithms demonstrated that the two clusters have strong stability (Figures 3B,C). The Kaplan-Meier method was used 


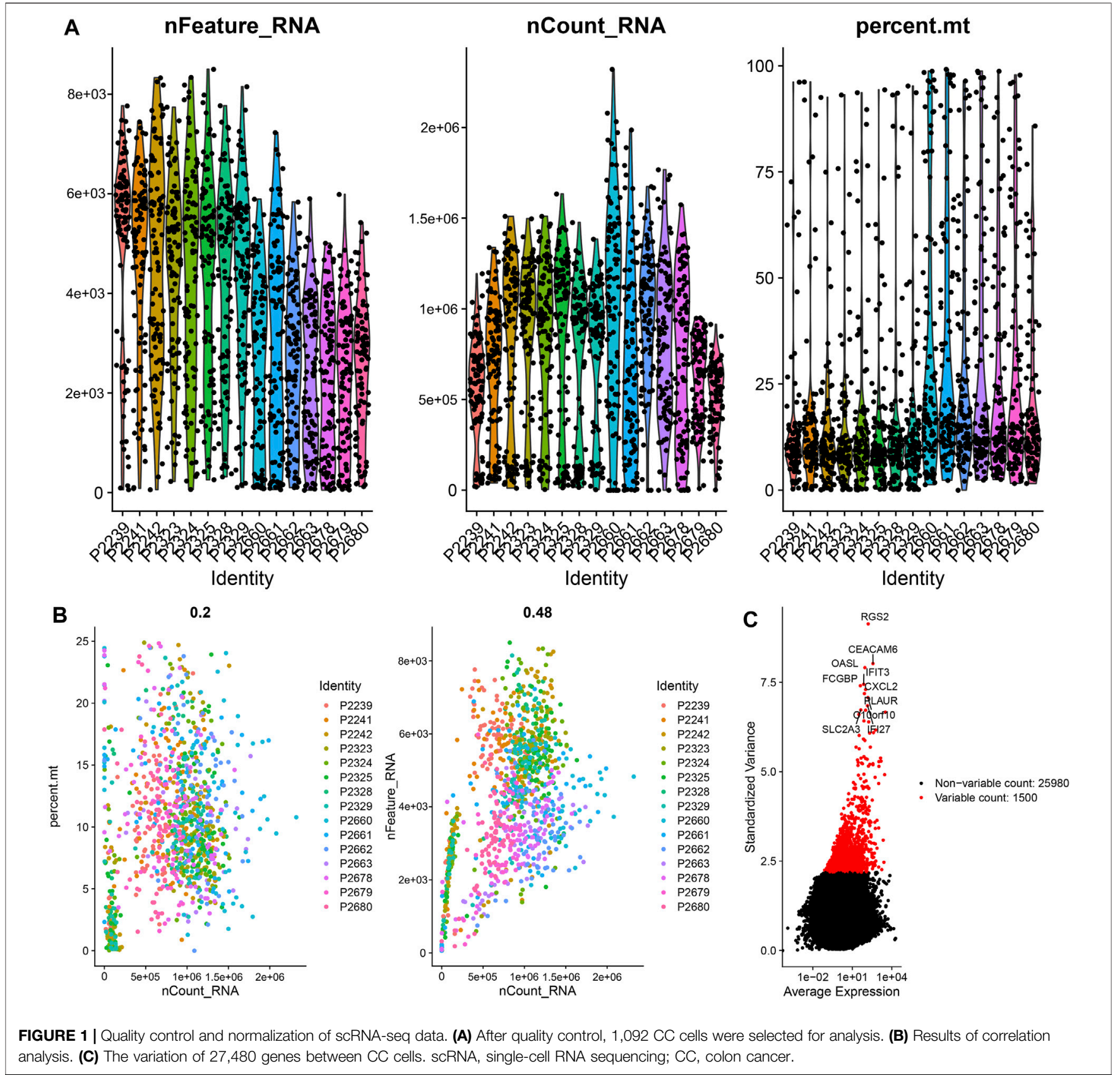

to determine the statistical significance of the consensus clustering results for $\mathrm{CC}$, and the results revealed that subtype 1 (C1) had better OS compared with that of subtype 2 (C2) (Figure 3D, $p=0.031$ ). Moreover, subtype 2 (C2) had more patients with a disease of advanced T stage and TNM stage (Figure 3E). Differentially expressed CDRGs between two molecular subtypes are displayed on a heat map (Figure 3F): The down/upregulated CDRGs in subtype 1 (C1) showed the same expression trend as that in subset II (branch II) (Figure 3G), whereas CDRGs in subtype 2 (C2) showed the same expression trend as that in subset I (branch I) (Figure 3H). These results demonstrated that subtype $1(\mathrm{C} 1)$ was mainly composed of CC cells in subset II (branch II), and subtype 2 (C2) was composed of CC cells in subset I (branch I).

\section{Integrated Analysis of Tumor Microenvironment Status and Immune Infiltration Between Two Molecular \\ Subtypes}

On the basis of the "ESTIMATE" package, the immune/stromal scores (all $p<0.001$ ) of subtype 2 (C2) were higher than those of subtype $1(\mathrm{C} 1)$ and the tumor purity $(p<0.001)$ of subtype $2(\mathrm{C} 2)$ was lower than that of subtype $1(\mathrm{C} 1)$ (Figures $4 \mathrm{~A}-\mathrm{C})$. The results of 


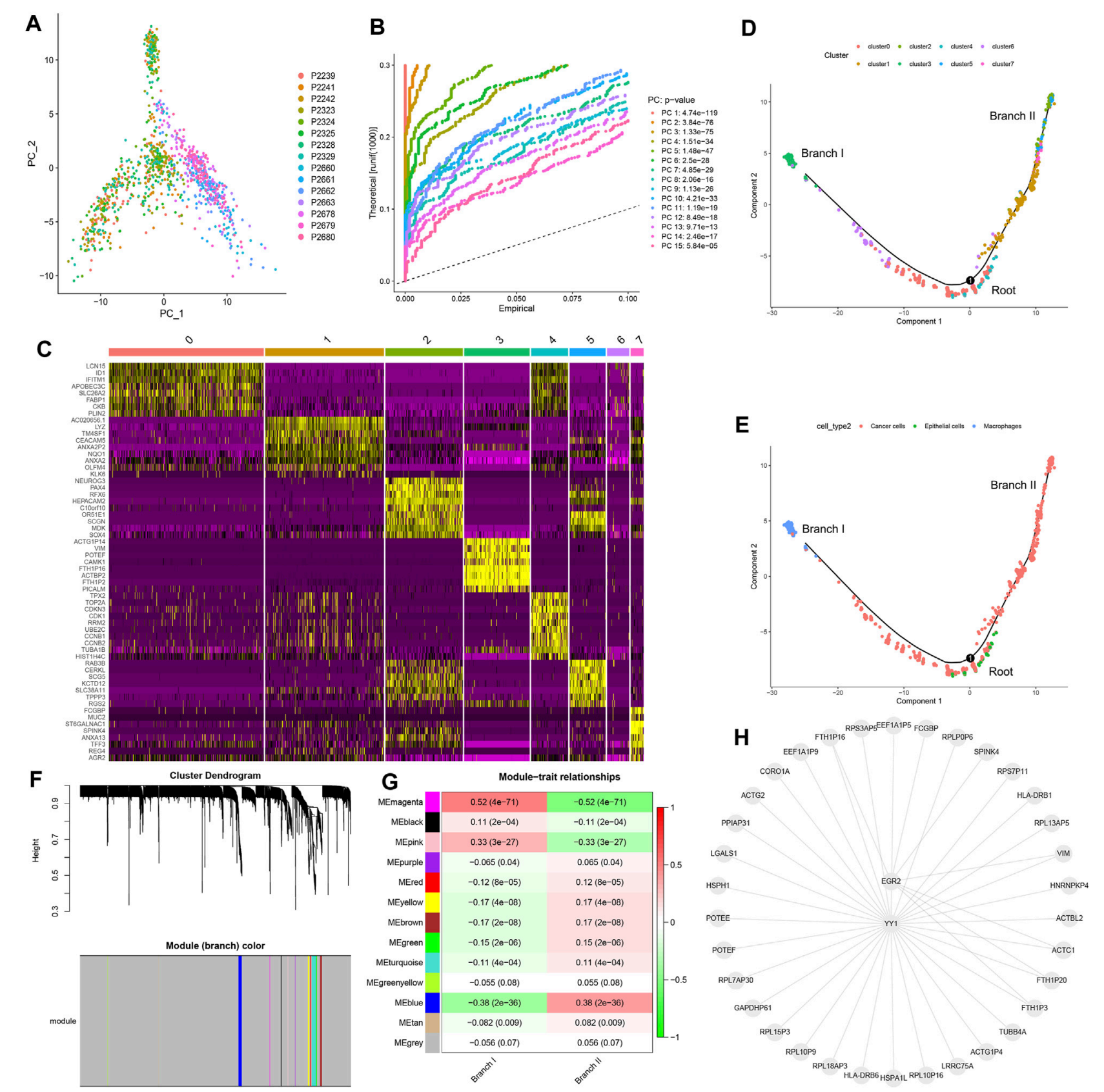

FIGURE 2 | Cell trajectory analysis and WGCNA. (A) PCA based on scRNA-seq data. (B) The top 15 PCs. (C) Eight clusters were identified by tSNE algorithm, and a heat map was plotted to represent marker genes (top 10\%) in each cluster. (D) Two subsets (namely, branch I and branch II) with distinct differentiation states were identified by cell trajectory analysis. (E) Cell types of subset I (branch I), subset II (branch II), and root. (F) Thirteen modules were identified using WGCNA. (G) Correlation analysis between modules and subsets. (H) Transcription factor enrichment analysis of key CDRGs. PCA, principal component analysis; PCs, principal components; tSNE, t-distributed stochastic neighbor embedding; CC, colon cancer; CDRG, CC differentiation-related gene. WGCNA, weighted correlation network analysis.

ssGSEA showed that the immune activity of subtype 2 (C2) was significantly higher than that of subtype 1 (C1) (Figure 4D), which was consistent with the immune score (Figure $4 \mathrm{~A}$ ). The infiltration density of 22 types of immune cells in each sample was calculated on the basis of the CIBERSORT algorithm, and the results were presented as different colors, which represented different cell types (Figure 4E). The results of differential analysis further demonstrated that subtype $1(\mathrm{C} 1)$ contained a higher distribution of memoryactivated $\mathrm{CD}^{+}{ }^{+} \mathrm{T}$ cells, plasma cells, follicular T helper cells, dendritic cells, and natural killer (NK) cells (Figure 4F, all $p<0.05$ ), whereas subtype 2 (C2) had a higher distribution of macrophages, neutrophils, and regulatory $\mathrm{T}$ cells (Tregs) (Figure 4G, all $p<0.05$ ). Together, subtype 2 (C2) with higher immune infiltration was associated with tumor immune escape mediated by Tregs and macrophages. 
A

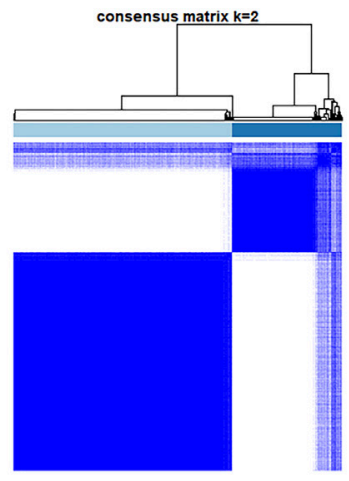

D

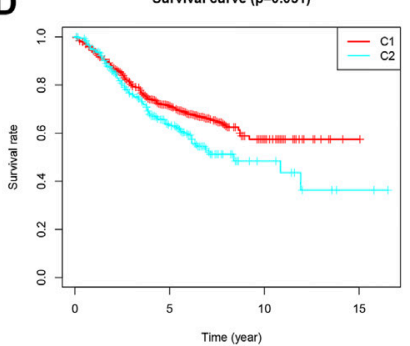

$\mathbf{F}$

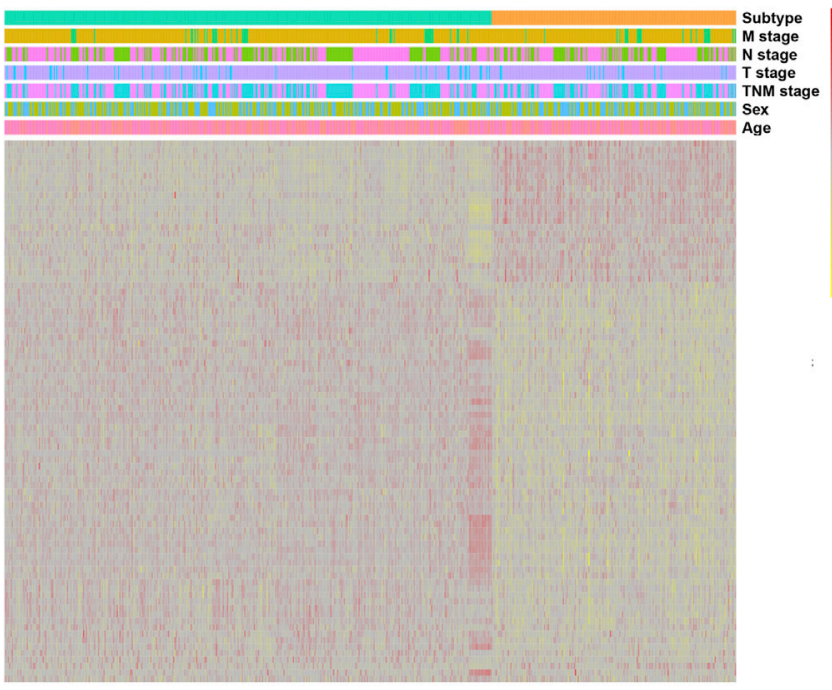

B
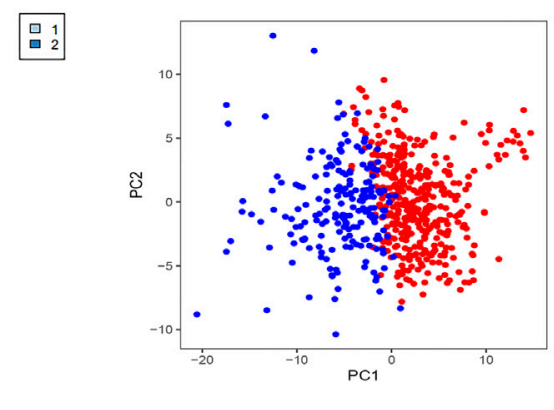

E
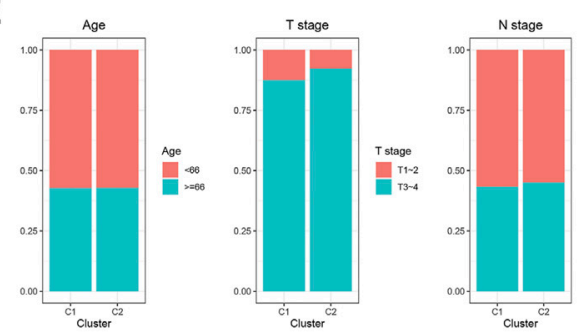

C

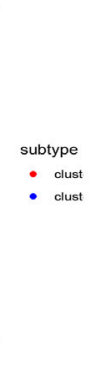

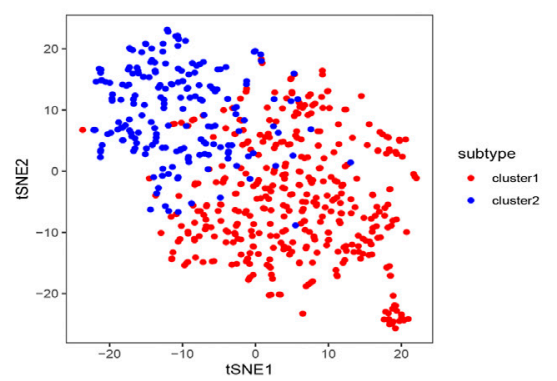

G
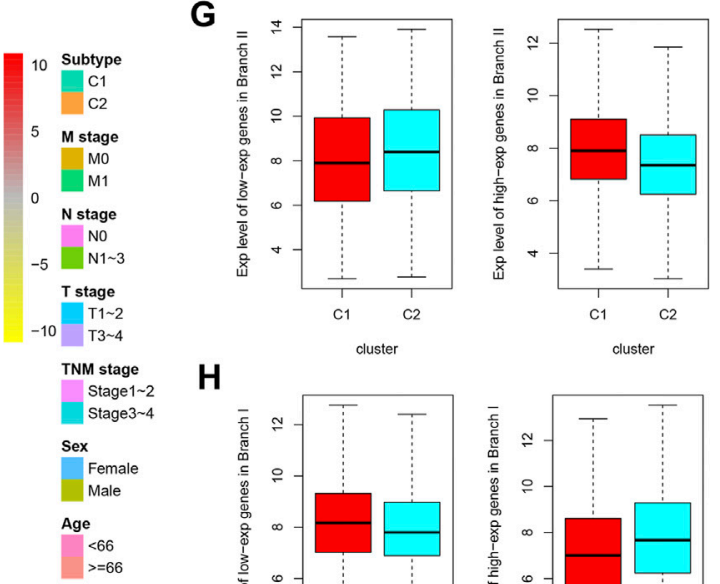

$\mathbf{H}$
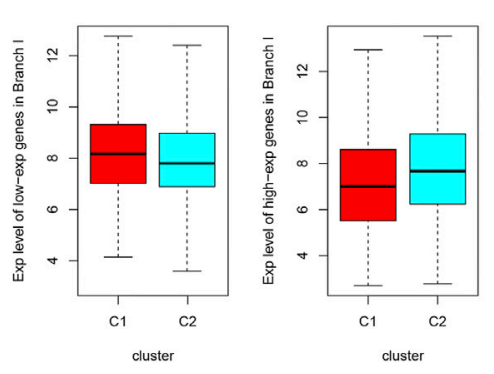

FIGURE 3 | CDRG-based consensus clustering analysis of CC patients from the GSE39582 dataset. (A-C) Two molecular subtypes were identified at a clustering threshold of maxK = 2. (B) PCA. (C) tSNE algorithm. (D) Kaplan-Meier analysis between subtype 1 (C1) and subtype 2 (C2). (E) The proportion of clinicopathological variables across the two subtypes. (F) A heat map of differentially expressed CDRGs among the two subtypes. (G) The down/upregulated CDRGs in subtype 1 (C1) showed the same expression trend as that in subset II (branch II). (H) The down/upregulated CDRGs in subtype 2 (C1) showed the same expression trend as that in subset I (branch I). CC, colon cancer; CDRGs, CC differentiation-related genes; PCA, principal component analysis; tSNE, t-distributed stochastic neighbor embedding.

However, subtype 2 (C2) lacked cytotoxic T lymphocytes, which are involved in anti-tumor immunity.

\section{Comparison of Expression Levels of ICGs Between Two Molecular Subtypes}

Thirty-seven confirmed ICGs were accessed from previous studies (Wu et al., 2016; Nishino et al., 2017; Patel et al., 2017;
Yang et al., 2017; Garris et al., 2018; Zhang et al., 2018; Wang et al., 2019a; Wang et al., 2019b; Han et al., 2019). Through differential expression analysis, we found 23 upregulated ICGs (B2M, CD28, CD40, CD80, CD86, CD8A, CTLA4, HAVCR2, ICOS, IFNG, IL23A, JAK2, LAG3, LDHA, LDHB, PDCD1LG2, PTPRC, PVR, TNFRSF18, TNFRSF4, TNFRSF9, TNFSF4, and TNFSF9) in subtype 2 (C2) (Figure 5A, all $p<0.05)$. More importantly, upregulated ICGs, including CD28 $(p<0.05)$, 

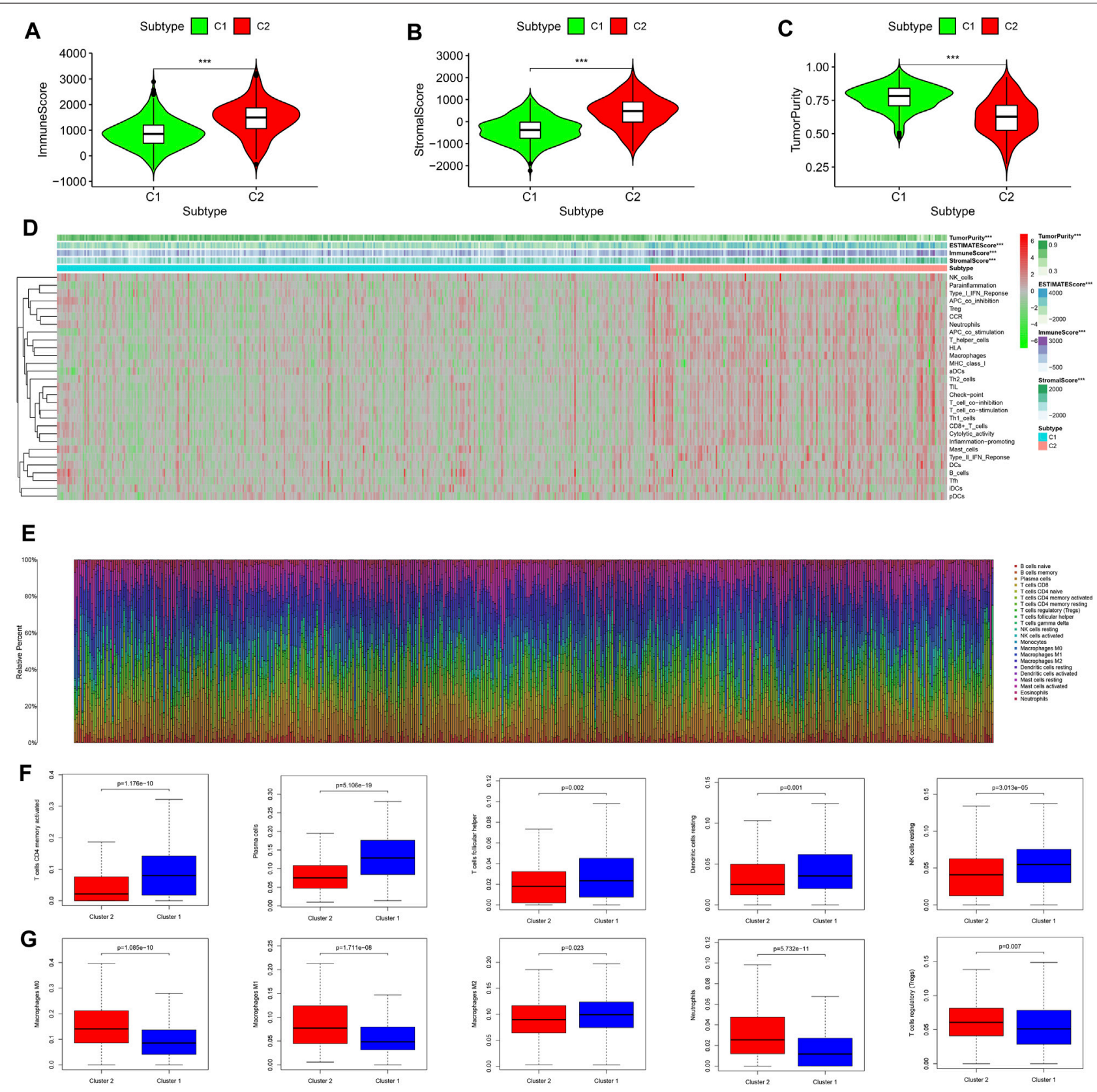

FIGURE 4 | Comprehensive analysis of the tumor microenvironment status and immune infiltration across two molecular subtypes. (A) Immune scores of the two molecular subtypes. (B) Stromal scores of the two molecular subtypes. (C) Tumor purity status of the two molecular subtypes. (D) Heat map of ssGSEA scores for the two molecular subtypes. (E) The infiltration density of 22 immune cells in each sample. (F) Immune cells with higher fraction in subtype 1 (C1). (G) Immune cells with higher fraction in subtype 2 (C2). ssGSEA, single-sample gene set enrichment analysis.

CTLA4 $(p<0.05)$, PVR $(p<0.05)$, CD80, CD86, IFNG, JAK2, LDHA, PTPRC, TNFRSF9, and TNFSF, were correlated with worse OS (Figure 5B). Only three ICGs (namely, CD40LG, LGALS9, and YTHDF1) were highly expressed in subtype 1 (C1) (Figure 5A, all $p<0.05$ ). Interestingly, upregulated LGALS9 was correlated with a better OS (Figure $5 \mathbf{B}$ ), whereas CD40LG and YTHDF1 were not associated with OS. These results indicate that upregulated ICGs are associated with a higher distribution of Tregs and adverse clinical outcomes of subtype
2 (C2) and are hence vital for guiding immunotherapy of CC subtypes.

\section{Differences in the Sensitivity of Two Molecular Subtypes to Chemotherapy}

Next, we estimated the IC50 of 12 chemotherapeutic agents in each sample from the GSE39582 dataset. Subtype 2 (C2) was more sensitive to camptothecin, mitomycin C, doxorubicin, 


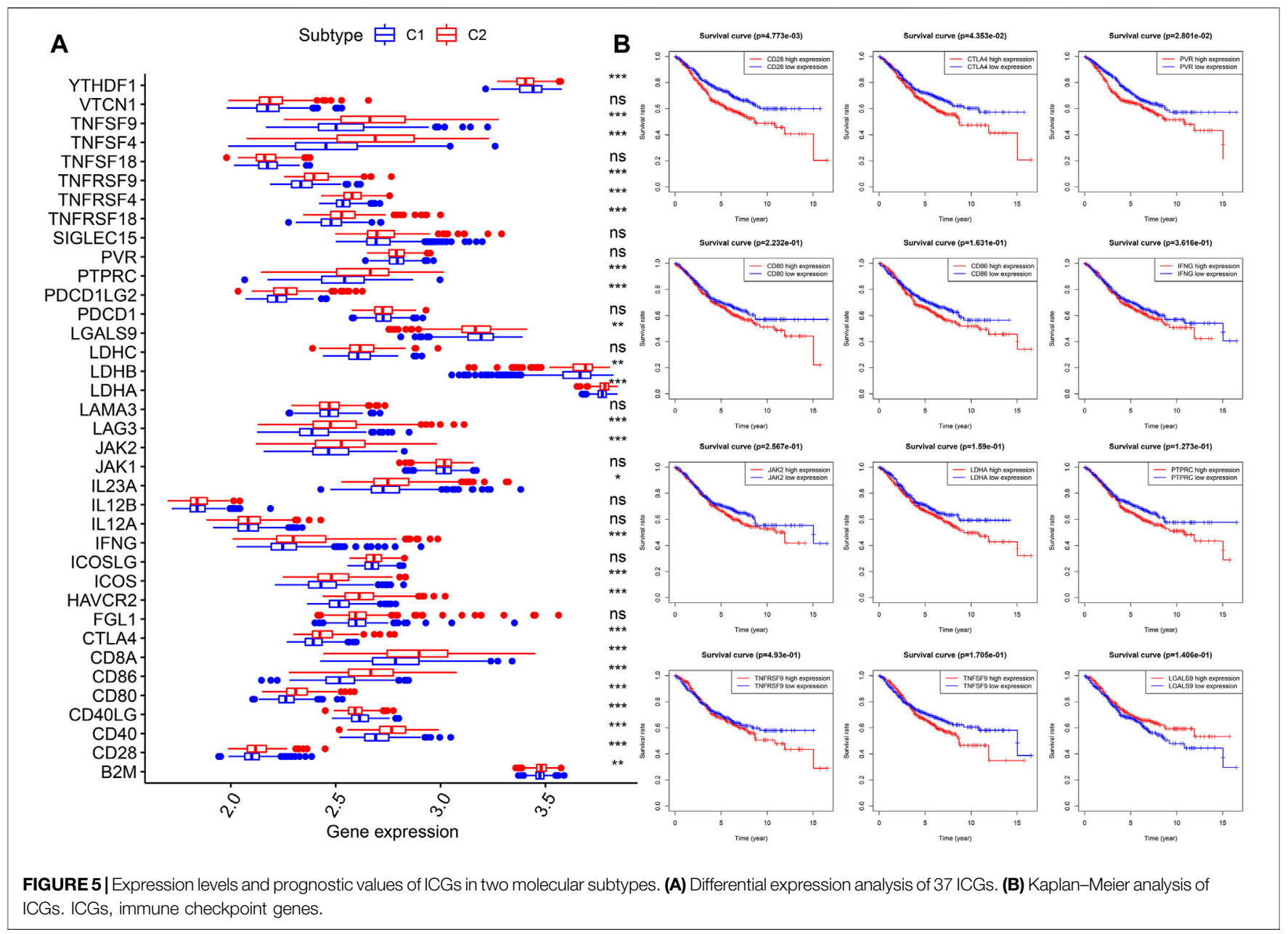

gemcitabine, paclitaxel, rapamycin, bleomycin, docetaxel, sunitinib, cisplatin, and vinblastine than subtype 1 (C1) (Figure 6A, all $p<0.001$ ). However, subtype 1 (C1) was more sensitive to sorafenib than subtype $2(\mathrm{C} 2)$ (Figure 6B, $p=0.013$ ).

\section{Construction, Evaluation, and Validation of a Five-CDRG-Based RS Signature}

After obtaining the intersection of CDRGs in TCGA and GSE39582 datasets, a total of 673 CDRGs were subjected to differential analysis, and 209 differentially expressed CDRGs were identified (Figure 7A). The results of univariate analysis further revealed 19 prognostic CDRGs (Figure 7B), which were enrolled into Lasso Cox regression analysis, and finally, a fiveCDRG-based RS signature was generated. The RS of each sample was calculated on the basis of the relative coefficient and expression levels of five genes using the following formula: $\mathrm{RS}=(-0.0427$ * expression of UGT2A3) $+(-0.0192$ * expression of $S R I)+(-0.0230$ * expression of MRPL37 $)+$ $(-0.0145 *$ expression of KPNA2) $+(-0.0184$ * expression of $A C A A 2)$. The RS of each CC sample was calculated in the training and validation cohorts, and the results revealed that the OS of the low-risk group was significantly better than that of the high-risk group (Figure 7C, TCGA: $p=2.576 \mathrm{e}-04$; Figure 7D, GSE39582: $p=3.48 \mathrm{e}-05)$. Moreover, the values of the areas under the ROC curves for predicting 1- and 2-year OS were 0.712 and 0.709 , respectively, in the training set (Figure 7E), and 0.621 and 0.624 , respectively, in the validation set (Figure $7 \mathbf{F}$ ).

\section{Expression Levels and Prognostic Values of Five CDRGs in the RS Signature}

The results of qPCR analysis showed that ACAA2 $(p=0.01), S R I$ $(p<0.001)$, and UGT2A3 $(p<0.001)$ were downregulated in CC samples, whereas KPNA2 $(p<0.001)$ and MRPL37 $(p<0.001)$ were upregulated in CC samples (Figure 7G). The results of immunohistochemical staining confirmed that ACAA2 was highly expressed in normal glandular cells and moderately expressed in CC cells (Supplementary Figure S4A). KPNA2 was expressed at low intensity in normal glandular cells, endothelial cells, and stromal cells and was highly expressed in CC cells (Supplementary Figure S4B). Moreover, MRPL37 was moderately expressed in normal glandular cells and highly expressed in CC cells (Supplementary Figure S4C). SRI was upregulated in normal glandular cells and endothelial cells and was downregulated in CC cells (Supplementary Figure S4D). 

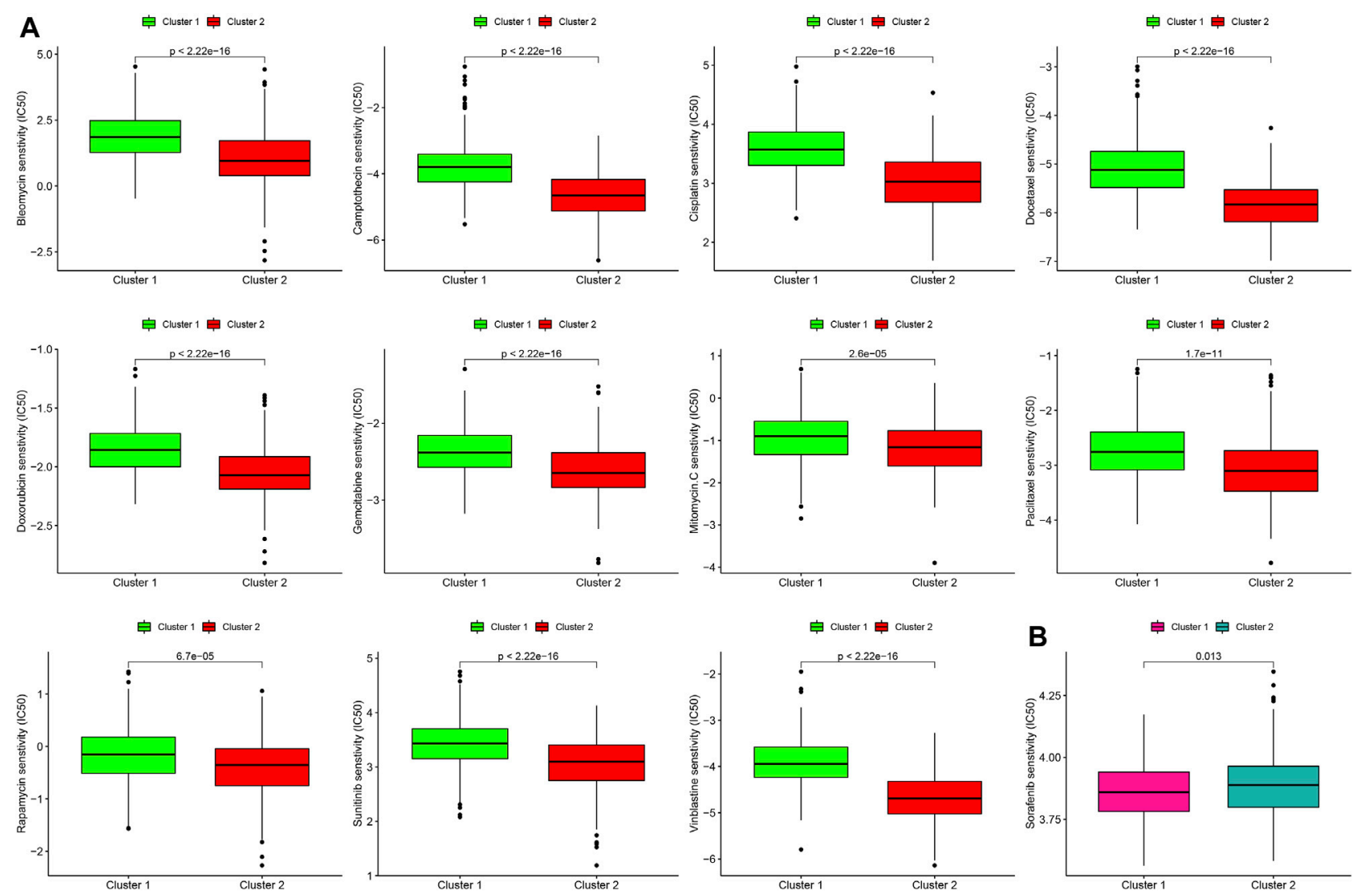

FIGURE 6 | Differences in the sensitivity of two molecular subtypes to chemotherapy. (A) Chemotherapeutic agents that CC cells in subtype 2 (C2) are more sensitive to. (B) Chemotherapeutic agents that CC cells in subtype 1 (C1) are more sensitive to.

The expression of UGT2A3 was higher in normal glandular cells and endothelial cells and was significantly lower in CC cells than that in normal cells (Supplementary Figure S4E). The scRNAseq data revealed that ACAA2, KPNA2, MRPL37, and SRI were downregulated in subset I (branch I, consisting of cluster 0/3/6), and UGT2A3 was downregulated in subset II (branch II, containing cluster 1/2/5/7) (Supplementary Figures S4F,G). The results of Kaplan-Meier analysis demonstrated that upregulation of five CDRGs was correlated with better OS rates (Supplementary Figure $\mathbf{S 4 H}$, all $p<0.05$ ).

\section{Construction, Evaluation, and Validation of a Nomogram for Predicting OS}

Univariate analysis was performed on the clinicopathological characteristics and RS in the training and validation cohorts, and the results revealed that patient age, TNM stage, T stage, $\mathrm{N}$ stage, $M$ stage, and RS jointly affected patient prognosis (TCGA: Figure 8A, all $p<0.05$; GSE39582: Figure 8B, all $p<0.05)$. Older age, later clinicopathological stage, and higher RS corresponded to poorer OS. Then, six prognostic factors were combined to establish a nomogram for predicting the OS of patients with CC on the basis of the training set (Figure 8C). The values of the areas under the ROC curves for predicting 3- and 5-year OS were 0.863 and 0.867 , respectively (Figure 8D), and the calibration curves for predicting 3- and 5-year OS were in good agreement with the actual observations (Figure 8D). In addition, the nomogram with the strongest predictive performance was verified in the training set (Figure 8E).

\section{DISCUSSION}

Previous research has highlighted the importance of tumor site, pathological type, early or advanced stage, therapy phase, and primary and metastatic focus in evaluating the therapeutic response and prognosis of patients with CC (Deng et al., 2006; Verhulst et al., 2012; Nitsche et al., 2013; Wang et al., 2016). Given the complexity of CC heterogeneity, the relationship between CC heterogeneity and clinical outcome and treatment response needs to be further studied. In the present study, we identified two CC subsets with distinct differentiation states, of which subset I (branch I) was related to hypoxia, metabolic disorders, and inflammatory responses, and subset II (branch II) was associated with immune responses and ferroptosis. CDRGbased consensus molecular classifications successfully predicted clinical outcomes, immune infiltration status, potential immunotherapy, and chemotherapeutic response in patients 
A
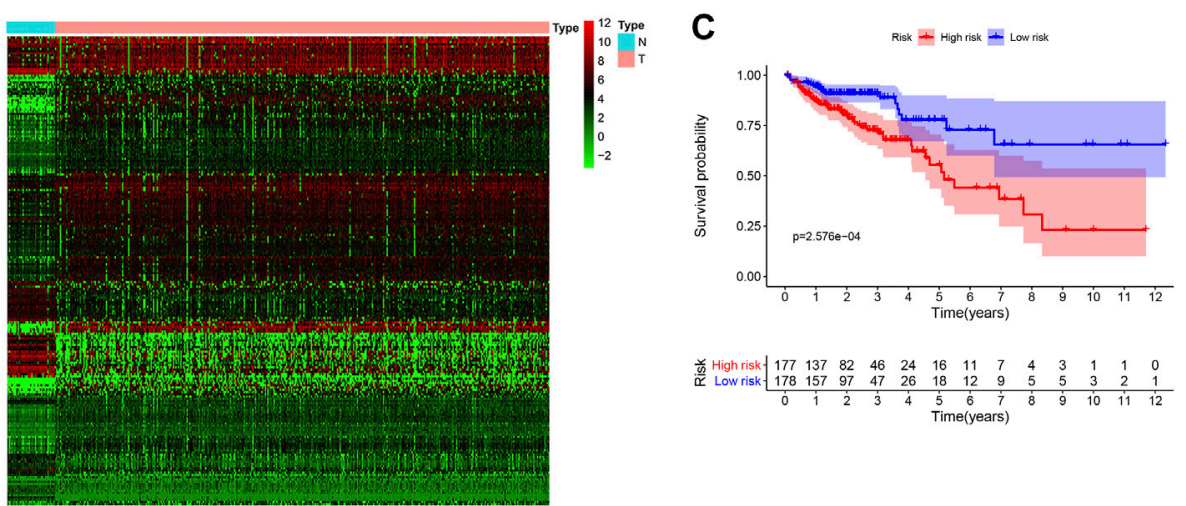

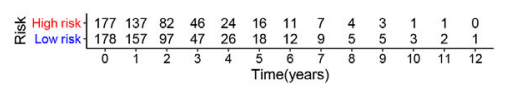

B
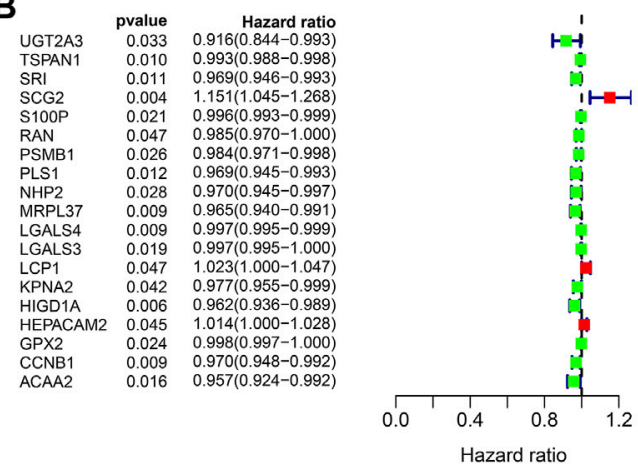

D

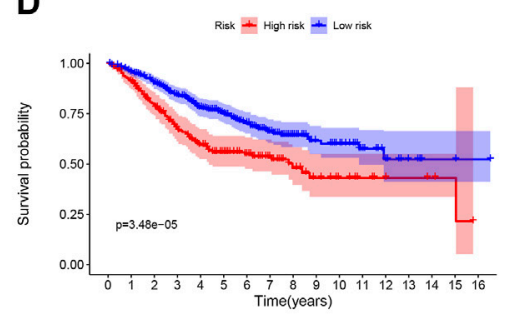

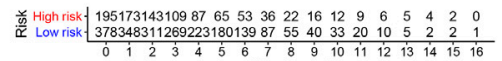

E

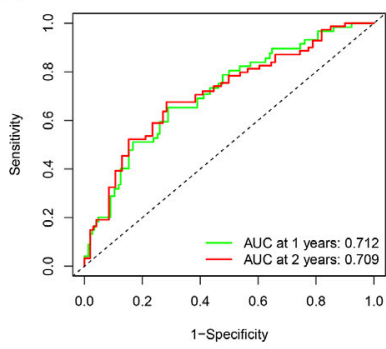

$\mathbf{F}$

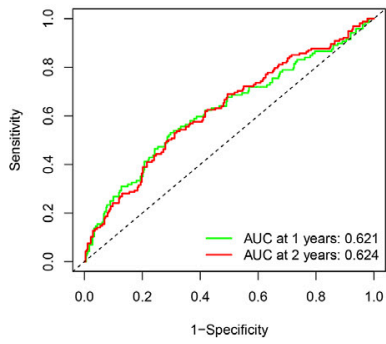

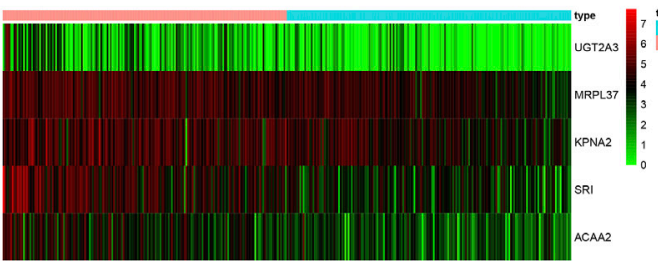
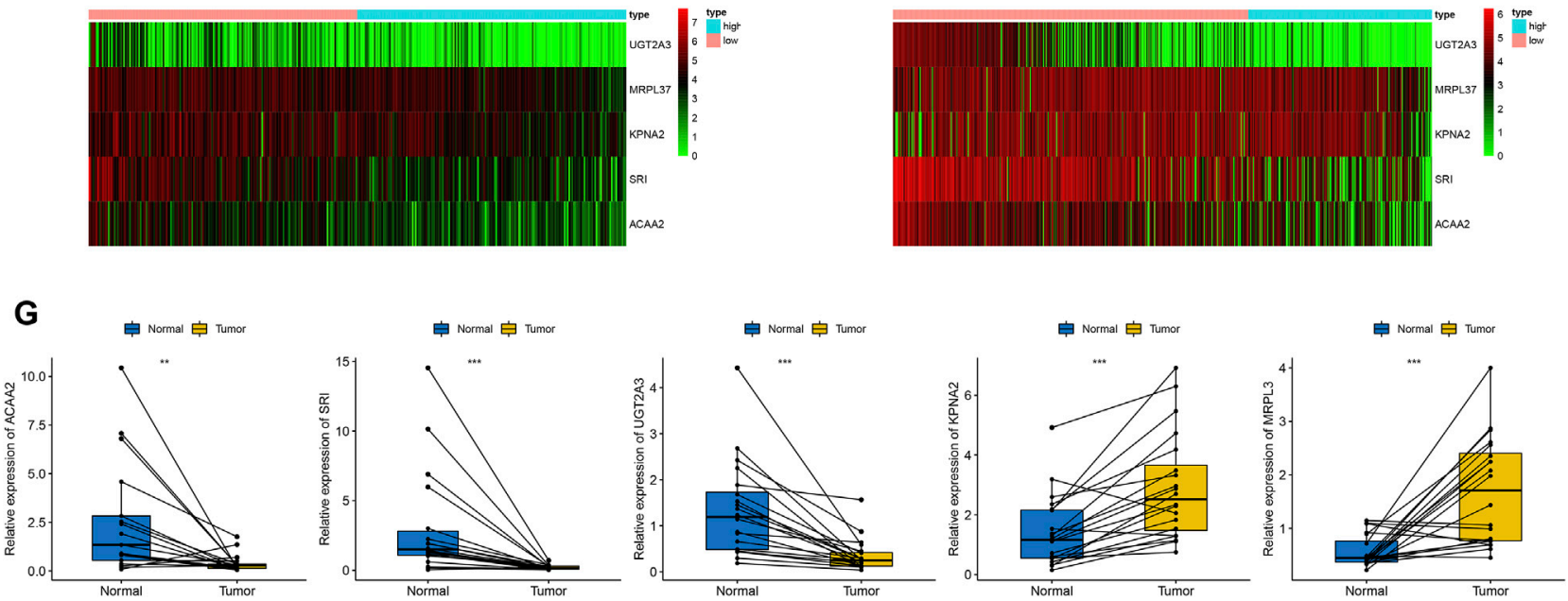

FIGURE 7 | Generation of a prognostic RS signature and quantitative PCR for CDRGs. (A) Differential expression of 209 CDRGs in normal tissues and CC samples with $|\log 2(F C)|>1$ and FDR $<0.05$. (B) Univariate analysis of CDRGs. Kaplan-Meier analysis between the low-risk group and the high-risk group in (C) the training set and (D) the validation set. The values of the areas under the ROC curves for predicting 3-, 4-, and 5-year OS in (E) the training set and (F) validation set. (G) Quantitative PCR for five CDRGs in the RS signature. ${ }^{*} p<0.05 ;{ }^{\star \star} p<0.01 ;{ }^{\star \star \star} p<0.001$. CC, colon cancer, CDRGs, CC differentiation-related genes, FDR, false discovery rate; OS, overall survival; ROC, receiver operating characteristic.

with CC. A nomogram combining the five-CDRG-based RS signature and prognostic clinicopathological characteristics accurately predicted the OS of patients with CC.

Gene-dependent intratumoral heterogeneity is an important factor that determines the biological behavior of CC cells. Different pathogeneses, natural evolution, and medical interventions produce a large number of molecular phenotypes, which can lead to differences in clinical features, therapeutic response, drug resistance, and prognosis among individuals with CC (Hanahan and Weinberg, 2011; DagogoJack and Shaw, 2018). In this study, CC cells with distinct differentiation states were projected into two subsets on the basis of cell trajectory analysis. These results demonstrate that CC cell differentiation may be related to the activation/inhibition 


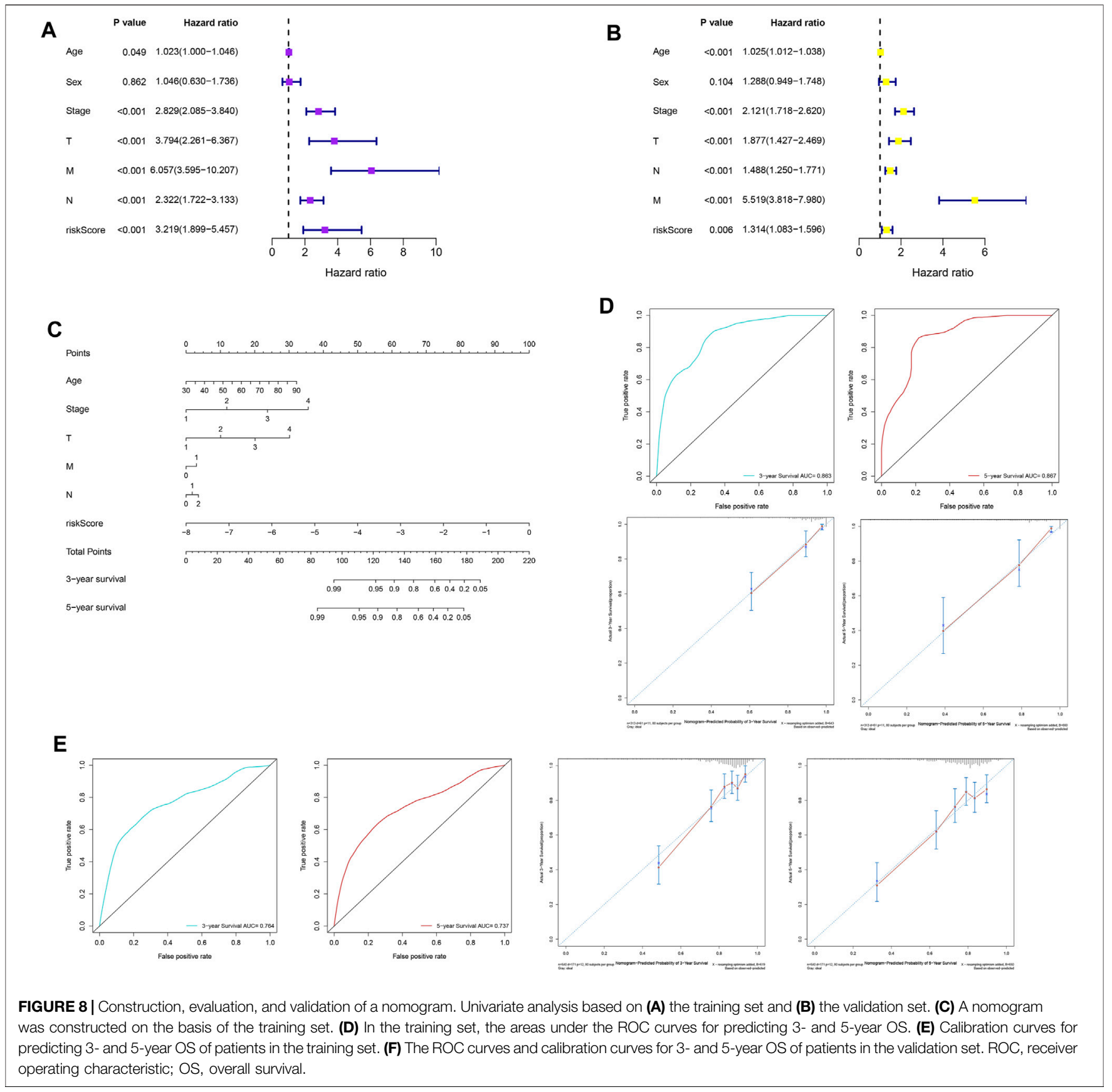

of various signaling pathways in a time- and dose-dependent manner. On the basis of WGCNA and TF enrichment analysis, we provided a benchmark for the investigation of intratumoral heterogeneity and the recognition of specific molecular phenotypes in CC.

Different molecular phenotypes and molecular types can guide disease diagnosis, optimize therapeutic strategies, and facilitate the development of new models for the advancement of precision medicine. In current studies, CC has been classified according to gene mutations, copy number, promoter methylation, noncoding RNA, microsatellite instability, and proteomics, and molecular subtypes of CC are based on tumor characteristics, therapeutic response, and prognosis, with an inconsistent pattern (Cancer Genome Atlas Network, 2012; Schlicker et al., 2012; Sinicrope and Sargent, 2012; Budinska et al., 2013; De Sousa E Melo et al., 2013; Marisa et al., 2013; Sadanandam et al., 2013; Roepman et al., 2014; Guinney et al., 2015). Therefore, it is worth exploring the possibility of introducing individualized treatment of CC based on molecular subtyping. In this study, CDRG-based molecular typing showed that the OS of subtype 1 (C1) was superior to that of subtype 2 (C2). Previous studies have shown that the infiltration of lymphocytes, such as $\mathrm{CD}^{+}{ }^{+} \mathrm{T}$ cells, NK cells, and plasma cells, was high in the tumor microenvironment (Almatroodi et al., 2016; Engblom et al., 2016; Jiang et al., 2016; 
Jain et al., 2017; Janakiram et al., 2017; Pierce et al., 2017; Song et al., 2017) and that the effectiveness of immunotherapy may be attributed to the upregulation of ICGs (e.g., programmed death-1, programmed death ligand-1, and cytotoxic $\mathrm{T}$ lymphocyte antigen-4) (Llosa et al., 2015). The infiltration density of memory-activated $\mathrm{CD}^{+} \mathrm{T}$ cells, follicular $\mathrm{T}$ helper cells, and NK cells was higher in subtype $1(\mathrm{C} 1)$ than in subtype $2(\mathrm{C} 2)$, and the proportion of macrophages and Tregs was lower in subtype 1 (C1) than in subtype 2 (C2). Moreover, the proportion of ICGs was higher in subtype $2(\mathrm{C} 2)$ than in subtype $\mathrm{C} 1(\mathrm{C} 1)$, suggesting that the subtype $\mathrm{C} 2(\mathrm{C} 2)$ tumors are characterized by immune escape and immunosuppression. Nevertheless, immune checkpoint inhibitors may reactivate the immune response of subtype 2 (C2) to exert anti-tumor effects (Riaz et al., 2017). Furthermore, patients with subtype 2 (C2) can be treated with several chemotherapeutic agents for improved prognosis.

The results of molecular subtyping revealed that patients with subtype $1(\mathrm{C} 1)$ had better survival outcomes, suggesting that the classification based on CDRGs can be used to predict the OS of patients with CC. Therefore, a CDRG-based RS signature with high accuracy and efficiency was established to evaluate the survival risk of patients with CC. To our knowledge, this is the first CDRG-based RS signature generated using Lasso Cox regression analysis, which can identify the gene combination with the best predictive ability. Moreover, a nomogram combining the CDRG-based RS and prognostic clinicopathological variables served as a visual model to predict patient OS, which was more accurate and effective than the RS signature.

There are some limitations to the current study. First, the prognostic RS signature was generated and verified on the basis of retrospective data from public open databases. Large-scale prospective clinical studies are needed to estimate the effectiveness and practicability of the RS signature. Second, although the nomogram for predicting OS has high predictive power, some clinicopathological parameters associated with prognosis are not available from the public open database, resulting in a limited number of variables involved in the nomogram. Therefore, it is necessary to further improve the nomogram in terms of higher predictive power and inclusion of more variables.

\section{CONCLUSION}

In this study, we identified two subsets with distinct differentiation states and revealed that CC molecular subtypes

\section{REFERENCES}

Almatroodi, S. A., McDonald, C. F., Darby, I. A., and Pouniotis, D. S. (2016). Characterization of M1/M2 Tumour-Associated Macrophages (TAMs) and Th1/Th2 Cytokine Profiles in Patients with NSCLC. Cancer Microenvironment 9, 1-11. doi:10.1007/s12307-015-0174-x

Bray, F., Ferlay, J., Soerjomataram, I., Siegel, R. L., Torre, L. A., and Jemal, A. (2018). Global Cancer Statistics 2018: GLOBOCAN Estimates of Incidence and Mortality Worldwide for 36 Cancers in 185 Countries. CA: A Cancer J. Clinicians 68, 394-424. doi:10.3322/caac.21492 on the basis of cell differentiation trajectories could successfully predict the patient prognosis, tumor microenvironment status, immune cell infiltration, and potential response to immunotherapy and chemotherapy. Moreover, the nomogram combining CDRG-based RS signature and clinicopathological characteristics accurately predicted the OS of patients with CC. Together, this study emphasizes the implications of CC cell differentiation for predicting prognosis and therapeutic response to immunotherapy and chemotherapy in patients and proposes a promising direction for clinical decision-making regarding CC treatment and prognosis.

\section{DATA AVAILABILITY STATEMENT}

The scRNA-seq data were accessed from the GSE148345 dataset (https://www.ncbi.nlm.nih.gov/geo/). The bulk RNA-seq data were obtained from the GSE39582 dataset (https://www.ncbi. nlm.nih.gov/geo/) and TCGA database (http://cancergenome. nih.gov/).

\section{AUTHOR CONTRIBUTIONS}

TF designed and supervised the project. RSX, JCF, YHG, and JR performed data analysis and wrote the paper. JCF completed qPCR analysis. RSX, JCF, and WS revised the manuscript.

\section{FUNDING}

This work was supported by the Talent Introduction Fund of Wuhan University Renmin Hospital (grant number NA to TF).

\section{ACKNOWLEDGMENTS}

The authors gratefully acknowledge each editor and reviewer for their profound insight into this study.

\section{SUPPLEMENTARY MATERIAL}

The Supplementary Material for this article can be found online at: https://www.frontiersin.org/articles/10.3389/fcell.2021.705537/ full\#supplementary-material

Budinska, E., Popovici, V., Tejpar, S., D’Ario, G., Lapique, N., Sikora, K. O., et al. (2013). Gene Expression Patterns Unveil a New Level of Molecular Heterogeneity in Colorectal Cancer. J. Pathol. 231, 63-76. doi:10.1002/path.4212

Cancer Genome Atlas Network (2012). Comprehensive Molecular Characterization of Human colon and Rectal Cancer. Nature 487, 330-337. doi:10.1038/nature11252 Dagogo-Jack, I., and Shaw, A. T. (2018). Tumour Heterogeneity and Resistance to Cancer Therapies. Nat. Rev. Clin. Oncol. 15, 81-94. doi:10.1038/nrclinonc.2017.166 De Sousa E Melo, F., Wang, X., Jansen, M., Fessler, E., Trinh, A., de Rooij, L. P. M. H., et al. (2013). Poor-Prognosis colon Cancer Is Defined by a Molecularly Distinct Subtype and Develops from Serrated Precursor Lesions. Nat. Med. 19, 614-618. doi:10.1038/nm.3174 
Deng, G., Nguyen, A., Tanaka, H., Matsuzaki, K., Bell, I., Mehta, K. R., et al. (2006). Regional Hypermethylation and Global Hypomethylation Are Associated with Altered Chromatin Conformation and Histone Acetylation in Colorectal Cancer. Int. J. Cancer 118, 2999-3005. doi:10.1002/ijc.21740

Engblom, C., Pfirschke, C., and Pittet, M. J. (2016). The Role of Myeloid Cells in Cancer Therapies. Nat. Rev. Cancer 16, 447-462. doi:10.1038/nrc.2016.54

Garris, C. S., Arlauckas, S. P., Kohler, R. H., Trefny, M. P., Garren, S., Piot, C., et al. (2018). Successful Anti-PD-1 Cancer Immunotherapy Requires T CellDendritic Cell Crosstalk Involving the Cytokines IFN- $\gamma$ and IL-12. Immunity 49, 1148-1161. e1147. doi:10.1016/j.immuni.2018.09.024

Guinney, J., Dienstmann, R., Wang, X., de Reyniès, A., Schlicker, A., Soneson, C., et al. (2015). The Consensus Molecular Subtypes of Colorectal Cancer. Nat. Med. 21, 1350-1356. doi:10.1038/nm.3967

Guo, Q., Cao, H., Qi, X., Li, H., Ye, P., Wang, Z., et al. (2017). Research Progress in Reversal of Tumor Multi-Drug Resistance via Natural Products. Anticancer. Agents Med. Chem. 17, 1466-1476. doi:10.2174/1871520617666171016105704

Han, D., Liu, J., Chen, C., Dong, L., Liu, Y., Chang, R., et al. (2019). Anti-tumour Immunity Controlled through mRNA m6A Methylation and YTHDF1 in Dendritic Cells. Nature 566, 270-274. doi:10.1038/s41586-019-0916-x

Hanahan, D., and Weinberg, R. A. (2011). Hallmarks of Cancer: the Next Generation. Cell 144, 646-674. doi:10.1016/j.cell.2011.02.013

Holohan, C., Van Schaeybroeck, S., Longley, D. B., and Johnston, P. G. (2013). Cancer Drug Resistance: An Evolving Paradigm. Nat. Rev. Cancer 13, 714-726. doi:10.1038/nrc3599

Jain, A., Zhang, Q., and Toh, H.-C. (2017). Awakening Immunity against Cancer: A 2017 Primer for Clinicians. Chin. J. Cancer 36, 67. doi:10.1186/s40880-017-0233-4

Janakiram, M., Shah, U. A., Liu, W., Zhao, A., Schoenberg, M. P., and Zang, X. (2017). The Third Group of the B7- CD 28 Immune Checkpoint Family: HHLA 2, TMIGD 2, B7x, and B7-H3. Immunol. Rev. 276, 26-39. doi:10.1111/imr.12521

Jiang, W., Chan, C. K., Weissman, I. L., Kim, B. Y. S., and Hahn, S. M. (2016). Immune Priming of the Tumor Microenvironment by Radiation. Trends Cancer 2, 638-645. doi:10.1016/j.trecan.2016.09.007

Lall, S., Sinha, D., Bandyopadhyay, S., and Sengupta, D. (2018). Structure-Aware Principal Component Analysis for Single-Cell RNA-Seq Data. J. Comput. Biol. 25, 1365-1373. doi:10.1089/cmb.2018.0027

Li, S., Lu, X., Chi, P., and Pan, J. (2013). Identification of HOXB8 and KLK11 Expression Levels as Potential Biomarkers to Predict the Effects of FOLFOX4 Chemotherapy. Future Oncol. 9, 727-736. doi:10.2217/fon.13.25

Liang, J., Cai, W., and Sun, Z. (2014). Single-Cell Sequencing Technologies: Current and Future. J. Genet. Genomics 41, 513-528. doi:10.1016/j.jgg.2014.09.005

Llosa, N. J., Cruise, M., Tam, A., Wicks, E. C., Hechenbleikner, E. M., Taube, J. M., et al. (2015). The Vigorous Immune Microenvironment of Microsatellite Instable colon Cancer Is Balanced by Multiple Counter-Inhibitory Checkpoints. Cancer Discov. 5, 43-51. doi:10.1158/2159-8290.CD-14-0863

Lovett, M. (2013). The Applications of Single-Cell Genomics. Hum. Mol. Genet. 22, R22-R26. doi:10.1093/hmg/ddt377

Marisa, L., de Reyniès, A., Duval, A., Selves, J., Gaub, M. P., Vescovo, L., et al. (2013). Gene Expression Classification of Colon Cancer into Molecular Subtypes: Characterization, Validation, and Prognostic Value. Plos. Med. 10, e1001453. doi:10.1371/journal.pmed.1001453

Nishino, M., Ramaiya, N. H., Hatabu, H., and Hodi, F. S. (2017). Monitoring Immune-Checkpoint Blockade: Response Evaluation and Biomarker Development. Nat. Rev. Clin. Oncol. 14, 655-668. doi:10.1038/nrclinonc.2017.88

Nitsche, U., Zimmermann, A., Späth, C., Müller, T., Maak, M., Schuster, T., et al. (2013). Mucinous and Signet-Ring Cell Colorectal Cancers Differ from Classical Adenocarcinomas in Tumor Biology and Prognosis. Ann. Surg. 258, 775-783. doi:10.1097/SLA.0b013e3182a69f7e

Pagès, F., Mlecnik, B., Marliot, F., Bindea, G., Ou, F. S., Bifulco, C., et al. (2018). International Validation of the Consensus Immunoscore for the Classification of colon Cancer: A Prognostic and Accuracy Study. Lancet 391, 2128-2139. doi:10.1016/s0140-6736(18)30789-x

Parkin, D. M. (2001). Global Cancer Statistics in the Year 2000. Lancet Oncol. 2, 533-543. doi:10.1016/S1470-2045(01)00486-7

Patel, S. J., Sanjana, N. E., Kishton, R. J., Eidizadeh, A., Vodnala, S. K., Cam, M., et al. (2017). Identification of Essential Genes for Cancer Immunotherapy. Nature 548, 537-542. doi:10.1038/nature23477

Pierce, B., Bole, I., Patel, V., and Brown, D. L. (2017). Clinical Outcomes of Remote Ischemic Preconditioning Prior to Cardiac Surgery: A Meta-Analysis of Randomized Controlled Trials. J. Am. Heart Assoc. 6, e004666. doi:10.1161/JAHA.116.004666
Qiu, X., Mao, Q., Tang, Y., Wang, L., Chawla, R., Pliner, H. A., et al. (2017). Reversed Graph Embedding Resolves Complex Single-Cell Trajectories. Nat. Methods 14, 979-982. doi:10.1038/nmeth.4402

Riaz, N., Havel, J. J., Makarov, V., Desrichard, A., Urba, W. J., Sims, J. S., et al. (2017). Tumor and Microenvironment Evolution during Immunotherapy with Nivolumab. Cell 171, 934-949. doi:10.1016/j.cell.2017.09.028

Roepman, P., Schlicker, A., Tabernero, J., Majewski, I., Tian, S., Moreno, V., et al. (2014). Colorectal Cancer Intrinsic Subtypes Predict Chemotherapy Benefit, Deficient Mismatch Repair and Epithelial-to-Mesenchymal Transition. Int. J. Cancer 134, 552-562. doi:10.1002/ijc.28387

Sadanandam, A., Lyssiotis, C. A., Homicsko, K., Collisson, E. A., Gibb, W. J., Wullschleger, S., et al. (2013). A Colorectal Cancer Classification System that Associates Cellular Phenotype and Responses to Therapy. Nat. Med. 19, 619-625. doi:10.1038/nm.3175

Satija, R., Farrell, J. A., Gennert, D., Schier, A. F., and Regev, A. (2015). Spatial Reconstruction of Single-Cell Gene Expression Data. Nat. Biotechnol. 33, 495-502. doi:10.1038/nbt.3192

Schlicker, A., Beran, G., Chresta, C. M., McWalter, G., Pritchard, A., Weston, S., et al. (2012). Subtypes of Primary Colorectal Tumors Correlate with Response to Targeted Treatment in Colorectal Cell Lines. BMC Med. Genomics. 5, 66. doi:10.1186/1755-8794-5-66

Sinicrope, F. A., and Sargent, D. J. (2012). Molecular Pathways: Microsatellite Instability in Colorectal Cancer: Prognostic, Predictive, and Therapeutic Implications. Clin. Cancer Res. 18, 1506-1512. doi:10.1158/1078-0432.ccr-11-1469

Song, S., Tan, J., Miao, Y., Li, M., and Zhang, Q. (2017). Crosstalk of Autophagy and Apoptosis: Involvement of the Dual Role of Autophagy under ER Stress. J. Cel. Physiol. 232, 2977-2984. doi:10.1002/jcp.25785

Verhulst, J., Ferdinande, L., Demetter, P., and Ceelen, W. (2012). Mucinous Subtype as Prognostic Factor in Colorectal Cancer: A Systematic Review and MetaAnalysis. J. Clin. Pathol. 65, 381-388. doi:10.1136/jclinpath-2011-200340

Wang, J., Sanmamed, M. F., Datar, I., Su, T. T., Ji, L., Sun, J., et al. (2019). Fibrinogen-like Protein 1 Is a Major Immune Inhibitory Ligand of LAG-3. Cell 176, 334-347. doi:10.1016/j.cell.2018.11.010

Wang, J., Sun, J., Liu, L. N., Flies, D. B., Nie, X., Toki, M., et al. (2019). Siglec-15 as an Immune Suppressor and Potential Target for Normalization Cancer Immunotherapy. Nat. Med. 25, 656-666. doi:10.1038/s41591-019-0374-x

Wang, R., Ma, X., Li, Y., He, Y., Huang, D., Cai, S., et al. (2016). The Characteristics and Prognostic Effect of E-Cadherin Expression in Colorectal Signet Ring Cell Carcinoma. Plos. One. 11, e0160527. doi:10.1371/journal.pone.0160527

Wilkerson, M. D., and Hayes, D. N. (2010). ConsensusClusterPlus: a Class Discovery Tool with Confidence Assessments and Item Tracking. Bioinformatics 26, 1572-1573. doi:10.1093/bioinformatics/btq170

Wu, L., Deng, W.-W., Yu, G.-T., Mao, L., Bu, L.-L., Ma, S.-R., et al. (2016). B7-H4 Expression Indicates Poor Prognosis of Oral Squamous Cell Carcinoma. Cancer Immunol. Immunother. 65, 1035-1045. doi:10.1007/s00262-016-1867-9

Yang, W., Pan, W., Chen, S., Trendel, N., Jiang, S., Xiao, F., et al. (2017). Dynamic Regulation of CD28 Conformation and Signaling by Charged Lipids and Ions. Nat. Struct. Mol. Biol. 24, 1081-1092. doi:10.1038/nsmb.3489

Zhang, Q., Bi, J., Zheng, X., Chen, Y., Wang, H., Wu, W., et al. (2018). Blockade of the Checkpoint Receptor TIGIT Prevents NK Cell Exhaustion and Elicits Potent AntiTumor Immunity. Nat. Immunol. 19, 723-732. doi:10.1038/s41590-018-0132-0

Conflict of Interest: The authors declare that the research was conducted in the absence of any commercial or financial relationships that could be construed as a potential conflict of interest.

Publisher's Note: All claims expressed in this article are solely those of the authors and do not necessarily represent those of their affiliated organizations or those of the publisher, the editors, and the reviewers. Any product that may be evaluated in this article, or claim that may be made by its manufacturer, is not guaranteed or endorsed by the publisher.

Copyright $\odot 2021$ Xiang, Fu, Ge, Ren, Song and Fu. This is an open-access article distributed under the terms of the Creative Commons Attribution License (CC BY). The use, distribution or reproduction in other forums is permitted, provided the original author(s) and the copyright owner(s) are credited and that the original publication in this journal is cited, in accordance with accepted academic practice. No use, distribution or reproduction is permitted which does not comply with these terms. 